\title{
El comportamiento del factor de necrosis tumoral alfa e interleucina 6 en lesiones de vías biliares postcolecistectomía
}

\author{
ALEJANDRA G. MIRANDA-DÍAZ1', JOSÉ M. HERMOSILLO-SANDOVAL², \\ GONZALO HUERTA-ROSAS, GERMÁN G. LÓPEZ-GUILLÉN², \\ ERNESTO G. CARDONA-MUÑOZ3, TRINIDAD GARCÍA-IGLESIAS ${ }^{3}$, \\ FERMÍN PACHECO-MOISÉS ${ }^{4}$, MARÍA DEL P. ALATORRE-CARRANZA ${ }^{3}$
}

\section{Tumor necrosis factor-alpha and interleukin-6 levels among patients suffering a bile duct injury during cholecystectomy}

Background: During cholecystectomy, the bile duct may be injured. When this complication occurs, Kupffer cells are activated and produce tumor necrosis factor alpha (TNF- $\alpha$ ) and interleukin-6 (IL6) to phagocyte toxic products Aim: To measure serum levels of TNF- $\alpha$ and IL-6 among patients that suffered a bile duct injury after a cholecystectomy. Patients and Methods: Serum levels of TNF- $\alpha$ and IL-6 were measured prior to the bile-enteric derivation and after one year of follow-up, in 31 patients that had a complete bile duct obstruction after open or laparoscopic cholecystectomy and in 5 healthy controls. Results: At baseline TNF- $\alpha$ levels in healthy subjects and patients with bile duct injury were 0 and $43.9 \pm 2.9 \mathrm{ng} / \mathrm{mL}$, respectively $(p<0.01)$. At one year of follow up, TNF- $\alpha$ became undetectable among patients. At baseline, the values for IL-6 among healthy controls and patients were $3.0 \pm 2.0$ and $72.0 \pm 94.7 \mathrm{pg} / \mathrm{mL}$ respectively, $(p<0,004)$. After one year of follow up, IL-6 levels decreased to $6.4 \pm 0.3 \mathrm{pg} / \mathrm{mL}$ among patients. Conclusions: TNF- $\alpha$ and IL-6 levels were elevated before bile-enteric derivation among patients with bile duct injury and became normal one year later.

(Rev Med Chile 2010; 138: 1259-1263).

Key words: Bile ducts, extrahepatic; Interleukin-6; Tumor necrosis factor-alpha.

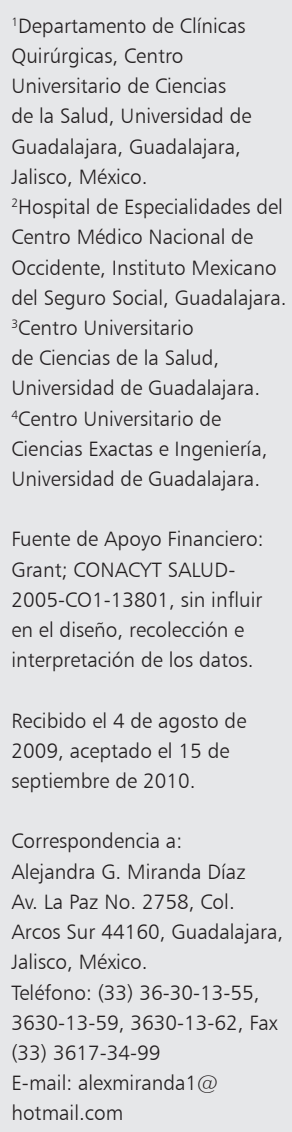

L as lesiones de vías biliares (LVB) post colecistectomía continúan siendo un problema mayor en la práctica quirúrgica ${ }^{1}$. Las LVB con obstrucción completa de las vías biliares se asocian con incremento de la morbilidad, disminución de la calidad de vida y pobre sobrevida ${ }^{2}$. El rango de incidencia de presentación de las LVB postcolecistectomía varía de 0,3 a $1,4 \%{ }^{3}$.

Cuando se presenta una lesión hepática cualquiera que sea la etiología, se activa la producción de citocinas que son mensajeros de bajo peso molecular secretadas por células que alteran el comportamiento de la misma célula (función autocrina) o a células vecinas (función paracrina), incluso a células de diferentes órganos (función endocrina $)^{4,5}$. Las citocinas son mediadores clave dentro del rol que juega el complejo entre las células inmunes y los hepatocitos mediante la activación de vías de señalización intracelular con el propósito de controlar la homeostasis celular hepática. Las células de Kupffer y los monocitos derivados de los macrófagos que infiltran el hígado ante cualquier lesión, son la fuente primaria de la producción de interleucina 1 alfa (IL- $1 \alpha$ ), del factor de necrosis tumoral alfa (TNF- $\alpha$ ) e interleucina 6 (IL-6) entre otras ${ }^{6,7}$. Las células de Kupffer son los 
macrófagos residentes del hígado que al activarse sintetizan TNF- $\alpha$ e IL-6 que se liberan directamente en el torrente circulatorio con el propósito de activar la fagocitosis de compuestos tóxicos. Las células de Kupffer pueden liberar diversas citocinas con función pro-inflamatoria como el TNF- $\alpha$ y la IL-6 y otras con función anti-inflamatoria como la interleucina 10 (IL-10), también promueven la activación de la cascada inflamatoria mediante la activación de linfocitos T, la inducción de apoptosis de los hepatocitos o la activación de las células estelares hepáticas fibrogénicas ${ }^{8}$.

El TNF- $\alpha$ es una citocina sintetizada por los macrófagos y neutrófilos, es esencial para la regeneración hepática mediante la activación del factor transcripcional $\mathrm{NF \kappa}^{9,10}$, con capacidad de activar vías pro-apoptóticas (principalmente caspasas) y anti-apoptóticas $(\mathrm{NF \kappa B})$ al mediar la respuesta inmune dentro del tracto portal en niños con atresia de vías biliares ${ }^{11} \mathrm{y}$ en adultos. $\mathrm{La}$ actividad inflamatoria que se presenta en las LVB podría semejar la cascada iniciada por la unión del TNF- $\alpha$ a los receptores TNF-R1 o TNF-RII ${ }^{12}$.

La IL-6 es una citocina cuyo promotor se asocia con receptores Janus cinasas (JAKs, JAK1, JAK2, y TYK) que se activan y fosforilan de residuos de tirosina en la molécula gp130. La IL-6 juega un importante rol en la señalización hepática de la respuesta inflamatoria aguda ${ }^{13}$ al participar en la patogénesis de la fibrosis hepática en pacientes con enfermedades hepáticas crónicas ${ }^{14}$. La expresión sérica aumentada se correlaciona con la progresión de la enfermedad ${ }^{15}$.

Aunque podría considerarse predecible encontrar aumentada la expresión hepática y sistémica del TNF- $\alpha$ e IL- 6 en pacientes con LVB, no se ha encontrado ningún reporte al respecto en la literatura. Nos planteamos como objetivo del estudio determinar el comportamiento sérico del TNF- $\alpha$ y la IL-6 en LVB postcolecistectomía inmediatamente previo a la reconstrucción bilio-digestiva y al año del postoperatorio.

\section{Pacientes y Métodos}

Se realizó un estudio transversal analítico, se formaron dos grupos de estudio:

Grupo 1: 5 sujetos sanos que sirvieron como control sano.

Grupo 2: 31 pacientes con LVB postcolecistec- tomía abierta o laparoscópica, con obstrucción completa de la vía biliar, enviados al Departamento de Cirugía General del Hospital de Especialidades del Centro Médico Nacional de Occidente del Instituto Mexicano del Seguro Social como hospital de referencia, para ser sometidos a la reconstrucción bilio-digestiva más conveniente dependiendo de las características clínicas de cada paciente. Se determinaron los niveles séricos de TNF- $\alpha$ e IL-6 inmediatamente antes de la reconstrucción quirúrgica y al año del postoperatorio. Se extrajo sangre periférica en ambos grupos.

Previo a la reconstrucción quirúrgica, los pacientes fueron sometidos a drenaje biliar externo. La reconstrucción bilio-digestiva se realizó en forma electiva a las 8 semanas en todos los casos. Las pruebas de coagulación fueron normalizadas. En quirófano se contó disponible sangre total, plasma fresco congelado y vitamina $\mathrm{K}$.

Veinte de los pacientes se enviaron a los hospitales de atención primaria para su seguimiento a largo plazo con instrucciones de ser regresados en caso de presentar complicaciones. Once de ellos con adscripción en la localidad se siguieron en el tiempo y se cuantificaron los niveles de TNF- $\alpha$ e IL-6 al año de la reconstrucción bilio-entérica

\section{ELISA de TNF- $\alpha$}

Se realizó la prueba comercial de ELISA (R\&D Systems) de acuerdo al protocolo del fabricante. Se preparó curva patrón de 8 puntos. Se pipeteó $900 \mu \mathrm{L}$ del diluyente dentro del tubo de 1.000 pg/ mL más $100 \mu \mathrm{L}$ del estándar, se pipeteó $500 \mu \mathrm{L}$ del diluyente calibrador dentro de los tubos y se agregó $500 \mu \mathrm{L}$ del estándar anterior para las diluciones seriales 1:2. El estándar no diluido sirvió como estándar mayor y el diluyente calibrador como estándar cero. Se adicionó $50 \mu \mathrm{L}$ del diluyente, luego $200 \mu \mathrm{L}$ del estándar, control o muestra en cada pozo. Se incubó 2 horas a temperatura ambiente, se lavó y se removió el buffer de acuerdo a cada tiempo, se adicionó $200 \mu \mathrm{L}$ del TNF- $\alpha$ conjugado y se incubó 2 horas, se adicionó $200 \mu \mathrm{L}$ de solución de substrato y se incubó 20 minutos a temperatura ambiente, se adicionó $50 \mu \mathrm{L}$ de solución de paro y se determinó la densidad óptica a $450 \mathrm{~nm}$.

\section{ELISA de IL-6}

Se realizó la prueba comercial de ELISA (R\&D Systems) de acuerdo al protocolo del fabricante. Se preparó curva patrón de 8 puntos. Se pipeteó 667 
$\mu \mathrm{L}$ del diluyente calibrador dentro del tubo de 100 $\mathrm{pg} / \mathrm{mL}$ más $500 \mu \mathrm{L}$ del diluyente dentro de cada tubo, se pipeteó $500 \mu \mathrm{L}$ del diluyente calibrador en los tubos restantes. El estándar no diluido sirvió como estándar mayor $(300 \mathrm{pg} / \mathrm{mL})$, el diluyente calibrador como estándar cero $(0 \mathrm{pg} / \mathrm{mL})$. Se adicionó $100 \mu \mathrm{L}$ del diluyente en cada pozo del estándar, control o muestra. Se incubó 2 horas a temperatura ambiente, se lavó y removió el buffer como se recomendó. Se adicionó $200 \mu \mathrm{L}$ de la IL-6 conjugada se incubó 2 horas. Se adicionó $200 \mu \mathrm{L}$ de solución de substrato y se incubó 20 minutos, se adicionó $50 \mu \mathrm{L}$ de solución de paro y se determinó la densidad óptica a $450 \mathrm{~nm}$.

\section{Análisis estadístico}

Los datos se expresan con medidas de tendencia central y de dispersión. Se utilizaron pruebas no paramétricas para las diferencias entre grupos. Se utilizó el programa SPSS para Windows (versión 10 , Chicago, IL). Se aceptó como significativa toda diferencia con valor $\mathrm{p} \leq 0,05$. El intervalo de confianza fue de $95 \%$.

\section{Consideraciones éticas}

El estudio se apegó a la declaración de Helsinki actualizada en el 2000, las leyes locales y nacionales. Se asignaron números de identificación para garantizar la confidencialidad de los pacientes. El protocolo y la forma de consentimiento informado fueron revisados y aprobados por el comité de ética e investigación del Hospital de Especialidades del Centro Médico Nacional de Occidente.

\section{Resultados}

\section{Manifestaciones Clínicas}

En el grupo de LVB se incluyeron 8 hombres y 23 mujeres con edad promedio de 47,44 $\pm 21,21$ años. La LVB se presentó en 16 colecistectomías abiertas y 15 laparoscópicas. Todos los pacientes fueron sometidos a nutrición artificial y antibióticos de amplio espectro antes de la cirugía para mejorar sus condiciones generales y minimizar los riesgos. La reconstrucción bilio-entérica se realizó en forma electiva en todos los casos por cirujanos con experiencia, de preferencia en el turno matutino. La media de tiempo quirúrgico fue de 6,5 horas. Los procedimientos quirúrgicos realizados fueron el Hepp-Couinaud eY de Roux.

\section{$T N F-\alpha$}

El resultado de la curva patrón de la prueba de ELISA obtuvo valor de $\mathrm{R}^{2}$ de 0,9997 . El promedio de los niveles séricos de TNF- $\alpha$ en los sujetos sanos fue de $0,0 \mathrm{ng} / \mathrm{mL}$, los pacientes con LVB obtuvieron 43,8901 $\pm 2,8808 \mathrm{ng} / \mathrm{mL}(\mathrm{p}<0,0001$ $\mathrm{U}$ de Mann-Whitney bilateral). Al año, los niveles del grupo con LVB se negativizaron 0,0505 \pm 0 . (Figura 1A). Si consideramos que el TNF- $\alpha$ es liberado en la circulación general y es sobre regulado a nivel sistémico en pacientes con sepsis, lo mismo podría suceder en los pacientes con LVB debido a que son portadores de colangitis por la obstrucción completa de las vías biliares. Todos los pacientes tuvieron material purulento en cavidad abdominal, bilis espesa o natas de fibrina que pudieron contribuir a la sobreexpresión del TNF- $\alpha$. $\mathrm{Al}$ año de la derivación bilio-digestiva, los niveles de TNF- $\alpha$ se negativizaron, lo que se podría traducir en la desaparición del estado inflamatorio.

\section{IL-6}

La curva patrón para la prueba de ELISA para IL-6 mostró valor de $\mathrm{R}^{2}$ de 0,9987 . Los niveles séricos de los sujetos sanos fue de 3,033 $\pm 1,990 \mathrm{pg} /$ $\mathrm{mL}$ y de los pacientes con LVB 71,973 \pm 94,691 pg/ $\mathrm{mL}$, más de 23 veces los valores control $(\mathrm{p}<0,004$ $\mathrm{U}$ de Mann-Whitney bilateral). Al año los niveles disminuyeron a $6,4463 \pm 0,2651 \mathrm{pg} / \mathrm{mL}$ (1B). Aunque la disminución fue significativa al año de seguimiento es el doble del valor de los sujetos sanos, lo que podría traducirse indirectamente en la presencia ya de fibrosis hepática.

La medición de ambas citocinas en el tiempo podría ser de utilidad en el seguimiento de los pacientes con LVB postcolecistectomía

\section{Discusión}

El comportamiento de las citocinas TNF- $\alpha$ e IL-6 estudiadas en los pacientes con LVB estuvieron significativamente sobre expresadas inmediatamente previo a la derivación bilio-entérica. Los valores séricos de las citocinas disminuyeron importantemente al año de seguimiento donde el TNF- $\alpha$ se negativizó completamente. Hay que considerar que el TNF- $\alpha$ es un potente estimulante de la reacción inflamatoria ${ }^{16}$ y podría participar en el proceso de fibrogénesis, aunque el rol del TNF- $\alpha$ en la modulación de la respuesta celular en rela- 

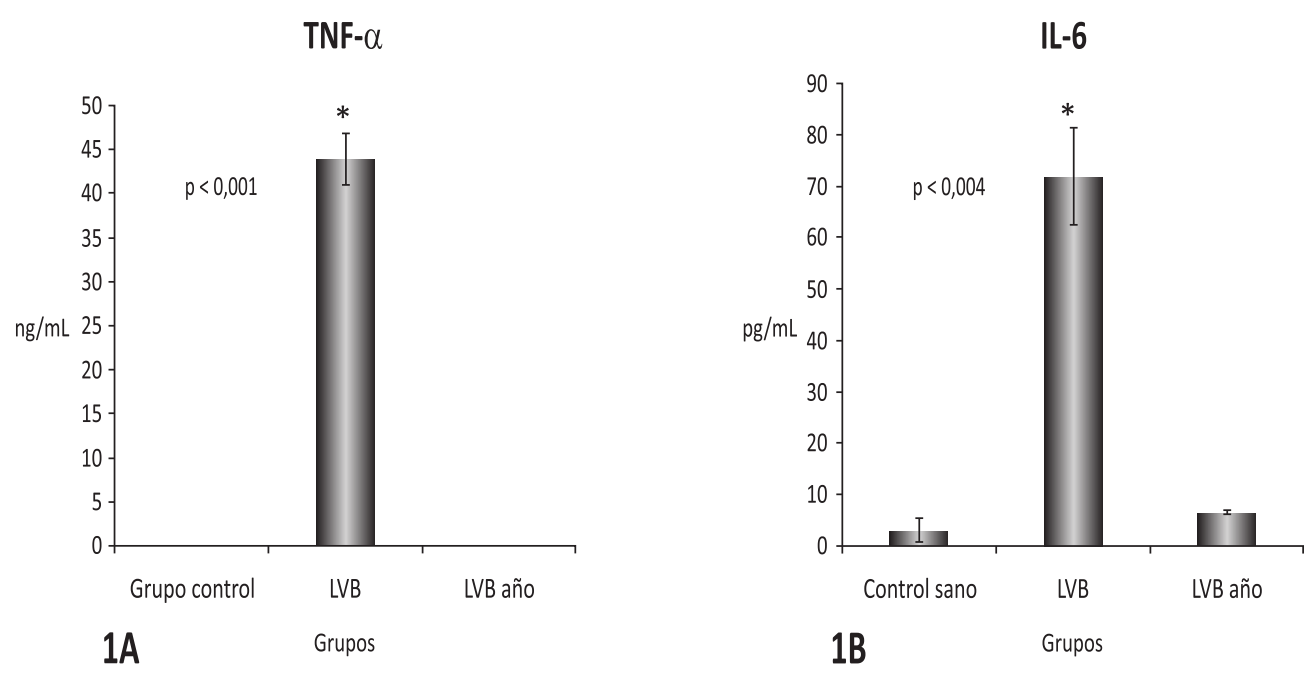

U de Mann-Whitney

Figura 1. Niveles séricos de TNF- $\alpha$ E IL-6. 1A). Se puede observar la importante elevación de los niveles séricos de TNF- $\alpha$ previo a la derivación bilio-entérica de los pacientes con LVB y la negativización al año de seguimiento. 1B) El comportamiento de la IL-6 fue significativamente similar con sobre expresión antes del procedimiento quirúrgico derivativo y la disminución importante al año de seguimiento aunque con persistencia de la producción mostrando el doble del nivel observado en los sujetos sanos, lo que podría estar en relación con el depósito de tejido fibroso en el hígado.

ción a inflamación y fibrosis en el tejido hepático lesionado no se ha dilucidado por completo ${ }^{17}$. Se considera que es capaz de inducir proliferación y activación de las células estelares hepáticas con formación de miofibroblastos/fibroblastos y contribuir en el desarrollo de fibrosis y remodelación hepática ${ }^{18}$.

Por otro lado, la IL-6 mostró comportamiento similar con 23 veces sobre expresión de la molécula y disminución importante al año de seguimiento de los pacientes, aunque sin llegar a los niveles control con persistencia del doble del observado en los sujetos control, situación que valdría la pena seguir su comportamiento por más tiempo.

Una de las explicaciones posibles para el comportamiento del TNF- $\alpha$ y de la IL-6 podría deberse a la respuesta inmune que se presenta en algunas colangiopatías como lo reportado para atresia de vías biliares en que las células del epitelio biliar son blanco de la respuesta inmune. El mecanismo efector responsable por el cual las células del epitelio biliar estimulan la respuesta inmune adaptativa en lesión del tracto biliar no está claro, pero podría deberse a la liberación de citocinas como las reportadas en el presente estudio. Otras moléculas que se sobre expresan y no se midieron en este estudio son las quemocinas que pueden perpetuar el daño del tracto biliar y producir necrosis o apoptosis de las células del epitelio biliar o de las células parenquimatosas ${ }^{19}$.

Las citocinas derivadas de los macrófagos como la IL-1, el TNF- $\alpha$ y la IL-6 juegan roles induciendo cambios que involucran diversas condiciones fisiopatológicas, no sólo reacciones inmunes e inflamatorias, también en la afectación general del organismo con desarrollo de caquexia que presentan los pacientes con $\mathrm{LVB}^{20}$. La activación y sobre expresión del TNF- $\alpha$ e IL-6 pueden deberse; a) la colecistectomía, b) la obstrucción completa de las vías biliares con estancamiento intra hepático de bilis, c) el efecto detergente persistente de los ácidos biliares con capacidad de producir daño hepático.

En concreto consideramos que la cuantificación periódica de los niveles de TNF- $\alpha$, IL-6, o ambos sería de utilidad en la detección de complicaciones en pacientes con LVB sometidos a reconstrucción bilio-digestiva. 
TNF- $\alpha$ e IL-6 post lesiones de vías biliares - A. G. Miranda-Díaz et al

Agradecimiento: A Rogelio Troyo Sanromán por su apoyo en el análisis estadístico.

\section{Referencias}

1. Flum DR, Cheadle A, Prela C. Bile duct injury during cholecystectomy and survival in medicare beneficiaries. JAMA 2003; 290: 2168-73.

2. Boerma D, Rauws EA, Keulemans YC. Impaired quality of life 5 years after bile duct injury during laparoscopic cholecystectomy: a prospective analysis. Ann Surg 2001; 234: 750-7.

3. Calvete J, Sabater L, Camps B. Bile duct injury during laparoscopic cholecystectomy: myth or reality of the learning curve? Surg Endosc 2000; 14: 608-11.

4. Luedde T, Liedtke C, Mamms MP, Trautwein C. Losing balance: cytokine signaling and cell death in the context of hepatocytes injury and hepatic failure. Eur Cytokine Netw 2002; 13: 377-83.

5. Decker K. biologically active products of stimulated liver macrophages (Kupffer cells). Eur J Biochem 1990; 192: 245-61.

6. Ramodori G, Armbrust T. Cytokines in the liver. Eur J Gastroenterol Hepatol 2001; 13: 777-84.

7. Ramadori G, Armbrust T. Cytokines and the liver. In: Oxford Textbook of Clinical Hepatology, 2nd edition. Bircher J, McIntyre N (editors). Oxford: Oxford University Press. 2000; 169-72.

8. Bilzer M, Roggel F, Gerbes AL. Role of Kupffer cells in host defense and liver disease. Liver Int 2006; 26: 1175 86.

9. Akerman P, Cote P, Yang SQ, McClain C, Nelson S, Bagby $\mathrm{G}$, et al. Antibodies to tumor necrosis factor- $\alpha$ inhibit liver regeneration after partial hepatectomy. Am J Phsiol 1992; 263: G579-85.

10. Plümpe J, Malek NP, Bock CT, Rakemann T, Manns MP, Trautwein C. NFאB determines between apoptosis and proliferation in hepatocytes during liver regeneration. Am J Physiol Gastrointest Liver Physiol 2000; 278: G17383.
11. Mack CL, Tucker RM, Sokol RJ, Karrer FM, Kotzin BL, Whitington PF, et al. Biliary atresia is associated with CD4+ Th1 cell-mediated portal tract inflammation. Pediatric Research 2004; 56: 79-87.

12. Kato A, Yoshidome H, Edwards MJ, Lentsch AB. Regulation of liver inflammatory injury by signal transducer and activator of transcription-6. Am J Pathol 2000; 157: 297-302.

13. Zhang D, Sun M, Samols D, Kushner I. STAT3 participates in transcriptional activation of the C-reactive protein gene by interleukin-6. J Biol Chem 1996; 271: 9503-9.

14. Toda K, Kumagai N, Tsuchimoto K, Inagaki H, Suzuki T, Oishi $\mathrm{T}$, et al. Induction of hepatic stellate cell proliferation by LPS-stimulated peripheral blood mononuclear cells from patients with liver cirrhosis. J Gastroenterol. 2000; 35: 214-20.

15. Streetz KL, Tacke F, Leifeld L, Wustefeld T, Graw A, Klein C, et al. Interleukin 6/gp 130-dependent pathways are protective during chronic liver disease. Hepatology 2003; 38: 218-29.

16. Bernard GR, Vincent JL, Laterre PF, LaRosa SP, Dhainaut JF, López-Rodríguez A, et al. Efficacy and safety of recombinant human activated protein $\mathrm{C}$ for severe sepsis. N Engl J Med 2001; 344: 699-709.

17. Lipsky PE, van der Heijde DM, St Clair EW, Furst DE, Breedveld FC, Kalden JR, et al. Infliximab and methotrexate in the treatment of rheumatoid arthritis. Antitumor Necrosis Factor Trial in Rheumatoid Arthritis with Concomitant Therapy Study Group. N Engl J Med 2000; 343: 1594-602.

18. Paulsson Y, Austgulen R, Hofsli E, Heldin CH, Westermark B, Nissen-Meyer J. Tumor necrosis factor-induced expression of platelet-derived growth factor a-chain messenger RNA in fibroblasts. Exp Cell Res 1989; 180: 490-96.

19. Lu BR, Mack CL. Inflammation and biliary tract inury. Curr Opin Gastroenterol 2009; 25(3): 260-4.

20. Argilés JM, López-Soriano FJ. Catabolic proinflammatory cytokines. Curr Opin Clin Nutr Metab Care 1998; 1: 24 . 